\title{
L'influence des modèles d'organisation de soins de santé primaires sur l'expérience de soins de patients atteints de différentes maladies chroniques
}

\author{
R. Pineault, M.D., Ph. D. (1,2,3); S. Provost, M.D., M. Sc. (1,2); M. Hamel, M. Sc. (1,2); A. Couture, M. Sc. (1,2);
}

J.-F. Levesque, M.D., Ph. D. $(1,2,3)$

\section{Résumé}

Objectifs : Déterminer dans quelle mesure l'expérience de soins varie selon les maladies chroniques et analyser la relation entre les modèles d'organisation de soins de santé primaires (SSP) et l'expérience de soins rapportée par des patients atteints de différentes maladies chroniques.

Méthodologie : Nous avons jumelé les résultats d'une enquête populationnelle et d'une enquête organisationnelle menées dans deux régions du Québec. Nous avons ciblé cinq groupes de maladies chroniques et les avons comparés à un groupe sans maladie chronique.

Résultats : L'accessibilité des soins est faible pour tous les groupes de maladies chroniques et présente peu de variation selon les maladies. Les modèles de contact et de coordination intégré sont les plus accessibles, tandis que le modèle à prestataire unique est le moins accessible. Les indices du processus et des résultats de soins sont beaucoup plus élevés que l'indice de l'accessibilité, et ce, pour tous les groupes. De plus, ils varient selon les maladies, les plus élevés se trouvant dans le groupe des facteurs de risque cardiovasculaires, et les plus faibles, dans le groupe des maladies respiratoires (patients âgés de 44 ans et moins). Toutefois, lorsqu'on passe du groupe des facteurs de risque aux groupes de maladies chroniques plus graves, on observe que le modèle de coordination intégré et le modèle communautaire affichent en général une meilleure performance quant au processus de soins, ce qui met en lumière le plus grand potentiel de ces deux modèles pour répondre aux besoins des personnes atteintes des maladies chroniques les plus graves dans le système de soins de santé canadien.

Mots clés : maladie chronique, modèles organisationnels, soins de santé primaires, continuité des soins, accessibilité des soins, Québec, processus de soins, résultats de soin

\section{Introduction}

Avec le vieillissement de nos populations, la prise en charge des maladies chroniques est devenue une préoccupation majeure des décideurs tout autant que des cliniciens ${ }^{1,2}$. Les systèmes de soins de santé doivent la prise en charge des patients plutôt que sur la prise en charge des maladies ${ }^{1,5,6}$. En fait, seulement $10 \%$ des malades chroniques ne sont atteints que d'une seule maladie, tandis que $60 \%$ présentent au moins quatre maladies $^{7}$. On peut donc soutenir que les soins de santé primaires offrent le contexte optimal pour la prise en charge des patients atteints de maladies chroniques ${ }^{4,8}$.

Dans plusieurs propositions, on a mis l'accent sur des approches de soins de santé primaires (SSP) qui préconisaient des soins axés sur le patient et qui soient à la fois plus accessibles et mieux coordonnés, soulignant par le fait même l'importance de la promotion de la santé et de la prévention des maladies ${ }^{9-11}$. Les modalités de soins comme celles proposées dans le « Chronic Care Model» et ses dérivés présentent un grand potentiel quant à l'atteinte de tels résultats ${ }^{12-14}$. Toutefois, on a moins porté attention au contexte organisationnel dans lequel ces modalités de soins sont mises en œuvre ${ }^{15}$. Ces modèles intégrés de soins aux malades chroniques ne précisent pas dans quel type d'organisation et selon quelles modalités organisationnelles de telles améliorations aux soins sont le plus susceptibles de se produire. Cependant, certains chercheurs ont exploré la relation entre les caractéristiques structurelles des types de pratique de SSP et leur performance, notamment l'expérience de soins ${ }^{14,16,17}$. Une étude effectuée récemment en Ontario a révélé que la prise en charge des maladies chroniques dans les centres de santé communautaire

Rattachement des auteurs

1. Direction de santé publique de l'Agence de la santé et des services sociaux de Montréal, Montréal (Québec), Canada

2. Institut national de santé publique du Québec, Québec (Québec), Canada

3. Centre de recherche du Centre hospitalier de l'Université de Montréal, Montréal (Québec), Canada

Correspondance : Raynald Pineault, Direction de santé publique, Agence de la santé et des services sociaux, 1301, rue Sherbrooke Est, Montréal (Québec) H2L 1M3;

tél. : 5145282400 poste 3480; téléc. : 514528 2470; courriel : rpineaul@santepub-mtl.qc.ca 
TABLEAU 1

Résultats de l'analyse factorielle de 23 variables de l'expérience de soins selon les répondants de l'enquête ( $\mathrm{N}=6 \mathbf{2 2 2})$ ayant une source habituelle de soins de santé primaires (SSP) (N = 473), Québec, 20032005

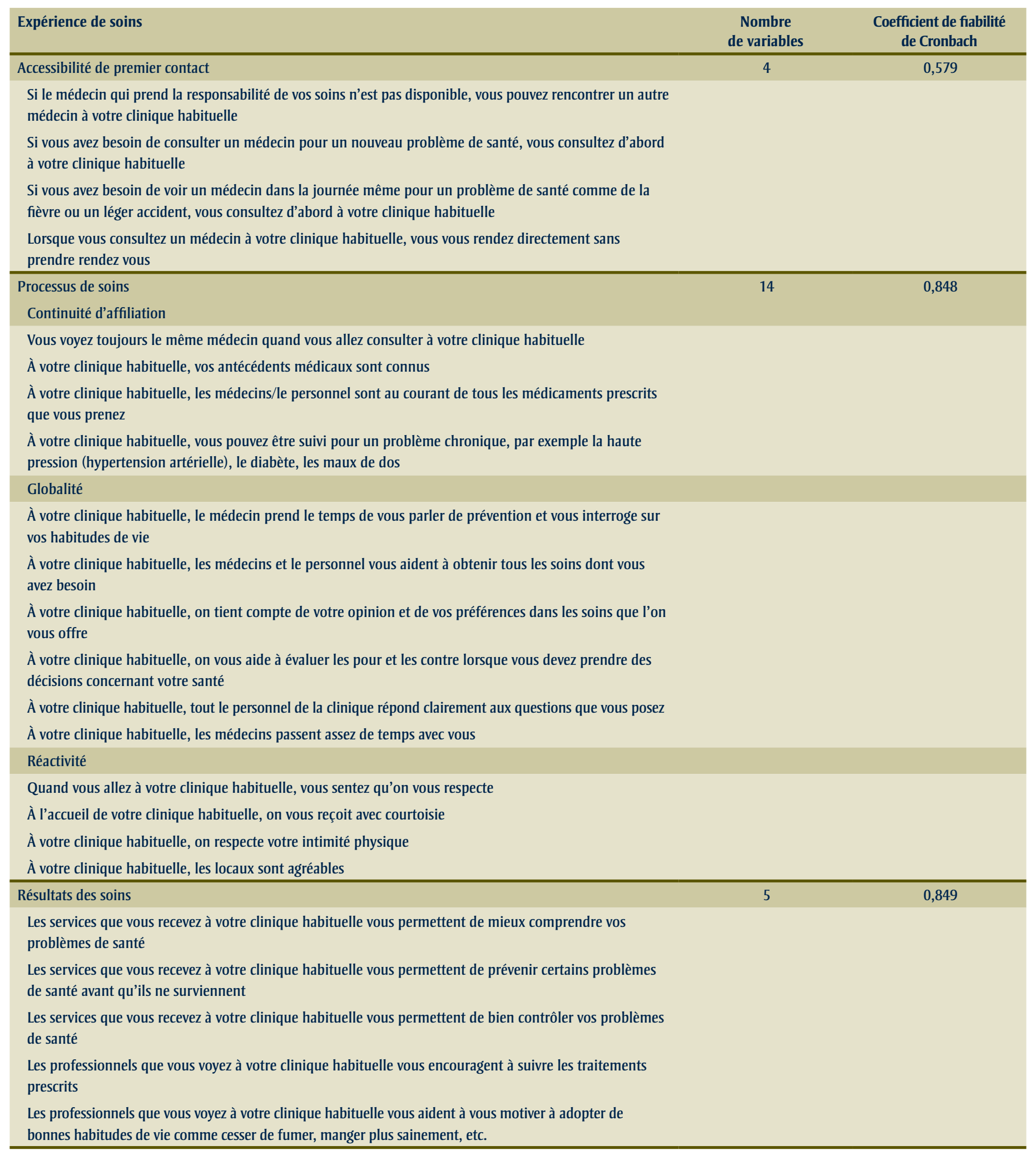


était supérieure à celle des autres types de pratique $^{18}$. Dans une autre étude ontarienne, on a comparé deux modèles de prestation de SSP ayant pour principale différence le mode de rémunération des médecins, soit le paiement à l'acte bonifié dans un cas, et le paiement par capitation dans l'autre ${ }^{19}$. Néanmoins, peu d'études ont examiné les types de pratique de SSP comme des entités organisationnelles complexes ${ }^{14,17,20,21}$. De plus, à notre connaissance, aucune étude n'a porté sur les variations de l'expérience de soins selon les différentes maladies chroniques et les divers modèles d'organisation de SSP.

Cet article vise à examiner dans quelle mesure l'expérience de soins varie selon les maladies chroniques et à analyser la relation entre les modèles d'organisation de SSP et l'expérience de soins rapportée par des patients atteints de diverses maladies chroniques.

\section{Méthodologie}

\section{Devis de recherche}

Notre étude consiste en deux enquêtes interreliées. La première, une enquête populationnelle, a été menée par téléphone en 2005 auprès de 9206 adultes (âgés de 18 ans ou plus) choisis au hasard dans deux régions $\mathrm{du}$ Québec. Pour veiller à ce que les 23 territoires soient localement représentés, l'échantillon a été stratifié de façon non proportionnelle. Par conséquent, toutes les analyses ont été effectuées à partir de données pondérées afin de tenir compte de cette caractéristique du plan d'échantillonnage. L'enquête a permis de documenter l'affiliation des répondants aux organisations de SSP, leur degré d'utilisation des services de santé, les attributs de leur expérience de soins et leur perception des besoins non comblés de soins de santé22,23.

Pour la deuxième enquête postale, 473 organisations de SSP situées dans les deux mêmes régions du Québec ont répondu. Cette enquête a permis d'évaluer les aspects liés à la vision, à la structure, aux ressources et aux pratiques des organisations de SSP. Dans chacune des organisations, un informateur clé, habituellement un médecin désigné par ses collègues, a répondu au questionnaire. Afin de permettre l'établissement d'une liaison nominale entre les deux enquêtes, on a demandé aux répondants de l'enquête populationnelle d'indiquer leur source habituelle de SSP.

Les taux de réponse ont atteint $64 \%$ dans le cas de l'enquête populationnelle et $75 \%$ dans le cas de l'enquête organisationnelle; $89 \%$ des répondants ont été jumelés à l'une des 473 organisations de SSP. Dans le cadre de cette étude, nous avons utilisé les réponses des 6222 répondants ayant eu recours aux services visés au cours des deux années précédant l'étude (2003-2005) et ayant comme source habituelle de soins l'une des 473 organisations de SSP. Des renseignements supplémentaires au sujet des enquêtes sont disponibles ailleurs ${ }^{22-24}$.

\section{Variables}

Pour les besoins de l'analyse, nous avons opérationnalisé deux concepts complexes : l'expérience de soins et le modèle d'organisation. À l'aide d'une analyse factorielle de 23 items tirés de l'enquête populationnelle, nous avons construit trois indices de l'expérience de soins : l'accessibilité de premier contact, le processus de soins et les résultats perçus des soins. L'accessibilité de premier contact correspond à la facilité avec laquelle les personnes peuvent avoir accès aux services de santé et utiliser ces services, tandis que le processus de soins correspond à la continuité d'affiliation et de suivi, à la globalité et à la réactivité. La continuité d'affiliation et de suivi se rapporte aux conditions associées au fait d'avoir une source régulière de soins et à la capacité de cette source à prendre en charge les maladies chroniques. La globalité mesure la capacité de l'organisation à répondre à un large éventail de besoins exprimés par le patient; la réactivité s'attache au respect et à l'attention accordée à dignité de la personne, ainsi qu'aux aspects non techniques des soins. Le tableau 1 présente les coefficients de fiabilité de Cronbach pour les trois indices de l'expérience de soins et pour les items qui composent ces indices.

Nous avons opérationnalisé les indices selon une approche qui mesure la performance en reclassant chaque item de catégories multiples en variables dichotomiques (inférieur/ supérieur $)^{25,26}$. Les réponses de la catégorie inférieure ont reçu la valeur 0 et celles de la catégorie supérieure ont reçu la valeur 1. Pour chaque indice, nous avons calculé la moyenne des scores dichotomisés et nous avons placé chaque indice sur une échelle de 0 à 10. Pour chacun des trois indices, nous avons créé une variable dichotomique en établissant un seuil de 7,5 fondé à la fois sur les distributions et sur le jugement d'un groupe de trois experts selon lequel un score de 7,5 ou plus représentait une meilleure performance, alors qu'un score de moins de 7,5 représentait une performance plus faible, mais pas nécessairement mauvaise. Nous avons ensuite analysé les trois variables dichotomiques suivantes : accessibilité de premier contact (élevée/ faible), processus de soins (élevé/faible) et résultats perçus des soins (élevés/faibles).

Nous avons conceptualisé les organisations selon quatre dimensions : la vision, les ressources, la structure et les pratiques ${ }^{27}$. La vision fait référence aux représentations, valeurs et orientations partagées par les membres de l'organisation. Les ressources correspondent au nombre de professionnels, à la quantité et au type de plateaux techniques et aux technologies des communications disponibles. La structure comprend les règles et le type de gouverne qui assurent la cohérence des organisations, sur le plan du fonctionnement interne aussi bien que des relations qu'elles entretiennent avec leur environnement. Finalement, les pratiques représentent les mécanismes cliniques et organisationnels qui soutendent la prestation des services ${ }^{27}$. En tout, nous avons associé 43 variables (décrites en détail ailleurs ${ }^{23}$ ) à ces dimensions. À partir de ces 43 variables, nous avons effectué une analyse de regroupement des 473 organisations de SSP et nous avons obtenu une taxonomie de cinq modèles organisationnels : un modèle communautaire et quatre modèles professionnels, à savoir les modèles à prestataire unique, de contact, de coordination et de coordination intégré. (Ces modèles ont été décrits plus en détail ailleurs ${ }^{23}$.)

Le tableau 2 présente les caractéristiques des quatre dimensions des modèles. Une lecture de gauche à droite du tableau 2 nous permet de constater que les modèles présentent des caractéristiques de plus en plus complexes, les modèles les plus complexes étant les modèles professionnel de coordination intégré et communautaire. La figure 1 présente la correspondance entre 
TABLEAU 2

Organisations de soins de santé primaires (SSP) étudiées ( $\mathrm{N}=473)$, Québec, 2003-2005

\begin{tabular}{|c|c|c|c|c|c|}
\hline \multirow[b]{2}{*}{ Caractéristiques } & \multicolumn{5}{|c|}{ Modèle professionnel de soins } \\
\hline & à prestataire unique & de contact & de coordination & de coordination intégré & communautaire \\
\hline \multicolumn{6}{|l|}{ Vision (valeurs) } \\
\hline Responsabilité & Clientèle $^{* *}$ & $\begin{array}{l}\text { Personnes qui se } \\
\text { présentent }\end{array}$ & Clientèle ${ }^{* * *}$ & Population ${ }^{* *}$ & Population ${ }^{* * *}$ \\
\hline Continuité - accessibilité & NS & $\begin{array}{l}\text { Accessibilité > } \\
\text { continuité }^{* * *}\end{array}$ & $\begin{array}{l}\text { Continuité > } \\
\text { accessibilité* }^{*}\end{array}$ & NS & $\begin{array}{l}\text { Continuité > } \\
\text { accessibilité }^{* *}\end{array}$ \\
\hline Travail d'équipe & Pas important ${ }^{* * *}$ & NS & Important ${ }^{* * *}$ & Important ${ }^{* * *}$ & Important ${ }^{* * *}$ \\
\hline \multicolumn{6}{|l|}{ Ressources } \\
\hline Présence de médecins & Peu $^{* * *}$ & Moyen* & Peu ${ }^{* * *}$ & Beaucoup ${ }^{* * *}$ & Beaucoup ${ }^{* * *}$ \\
\hline $\begin{array}{l}\text { Présence de } \\
\text { professionnels }\end{array}$ & Peu ${ }^{* * *}$ & Beaucoup ${ }^{* * *}$ & Beaucoup ${ }^{* * *}$ & Beaucoup *** & Moyen ${ }^{* * *}$ \\
\hline Plateaux techniques & Très peu ${ }^{* * *}$ & NS & Moyen ${ }^{* *}$ & Beaucoup ${ }^{* * *}$ & Moyen $^{* * *}$ \\
\hline $\begin{array}{l}\text { Technologies de } \\
\text { l'information et des } \\
\text { communications }\end{array}$ & Très peu ${ }^{* * *}$ & NS & NS & Beaucoup ${ }^{* * *}$ & Beaucoup ${ }^{* * *}$ \\
\hline \multicolumn{6}{|l|}{ Structure } \\
\hline Gouverne & Prof. privée ${ }^{* * *}$ & Prof. privée ${ }^{* * *}$ & Prof. privée ${ }^{* * *}$ & Prof. privée ${ }^{* * *}$ & Publique *** \\
\hline $\begin{array}{l}\text { Rémunération des } \\
\text { médecins }\end{array}$ & À l'acte ${ }^{* * *}$ & À l'acte ${ }^{* * *}$ & À l'acte ${ }^{* * *}$ & À l'acte ${ }^{* * *}$ & En fonction du temps ${ }^{* * *}$ \\
\hline Collaboration à l'interne & Aucune ${ }^{* * *}$ & Non structurée ${ }^{* *}$ & Non structurée ${ }^{* * *}$ & Structurée ${ }^{* * *}$ & Structurée ${ }^{* * *}$ \\
\hline $\begin{array}{l}\text { Lien avec les soins } \\
\text { primaires }\end{array}$ & NS & Non ${ }^{* *}$ & Non ${ }^{*}$ & Oui ${ }^{* * *}$ & NS \\
\hline $\begin{array}{l}\text { Lien avec les services } \\
\text { spécialisés }\end{array}$ & NS & Non ${ }^{*}$ & Non ${ }^{* *}$ & Oui ${ }^{* * *}$ & NS \\
\hline \multicolumn{6}{|l|}{ Pratiques } \\
\hline Avec/sans rendez-vous & $\begin{array}{l}\text { Surtout sur } \\
\text { rendez-vouss** }\end{array}$ & $\begin{array}{l}\text { Surtout sans } \\
\text { rendez-vous }\end{array}$ & NS & NS & NS \\
\hline Étendue des services & Restreinte $^{* * *}$ & Restreinte $^{* *}$ & Grande *** & Très grande ${ }^{* * *}$ & Très grande ${ }^{* * *}$ \\
\hline Évaluation de la qualité & Aucune ${ }^{* * *}$ & Plus ou moins $s^{* * *}$ & Plus ou moins *** & Plus *** & Plus *** \\
\hline
\end{tabular}

Abréviations : N, taille totale de l'échantillon; NS, non significatif; $p$, signification statistique; prof., professionnelle

Différence de degré des caractéristiques au sein de chaque modèle.

${ }^{*} p \leq 0,05$

${ }^{* *} p \leq 0,01$

**** $p \leq 0,001$

les types d'organisations de SSP qui existent actuellement dans les deux régions visées et les cinq modèles de la taxonomie.

\section{Sélection des maladies}

Comme nous l'avons déjà mentionné, nous avons utilisé les réponses des répondants qui avaient eu recours aux services visés au cours des deux années précédant l'étude (2003-2005) et qui avaient comme source habituelle de soins l'une des 473 organisations de SSP ( $\mathrm{N}=6$ 222). Nous avons interrogé les répondants sur leur expérience de soins et leur avons demandé si un médecin leur avait déjà dit qu'ils étaient atteints d'une ou de plusieurs des maladies chroniques énumérées dans le questionnaire*. Les répondants étaient ensuite classés en fonction de la présence ou non d'une maladie chronique. Les personnes qui étaient atteintes d'une seule maladie étaient classées dans la catégorie correspondant à cette maladie. Celles qui étaient atteintes de plusieurs maladies chroniques étaient classées dans la première catégorie figurant dans la liste de maladies triées selon un ordre décroissant, comme l'indique le diagramme présenté dans la figure 2 . Pour veiller à ce que le groupe sans maladie chronique $(37,6 \%)$

* Problèmes cardiaques (maladie cardiaque ou insuffisance cardiaque), respiratoires (maladie pulmonaire obstructive chronique [MPOC], asthme), arthritiques

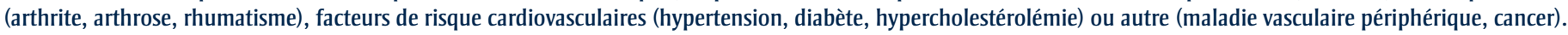


FIGURE 1

Correspondance entre les types d'organisations de soins de santé primaires (SSP) et les cinq modèles organisationnels, Québec, $20032005^{a}$

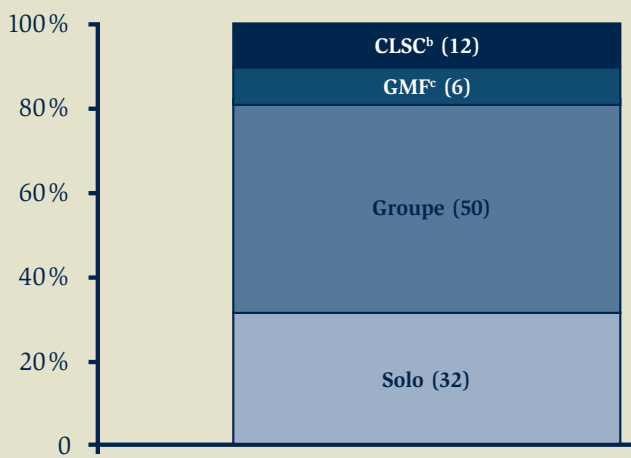

Types d'organisations de SSP

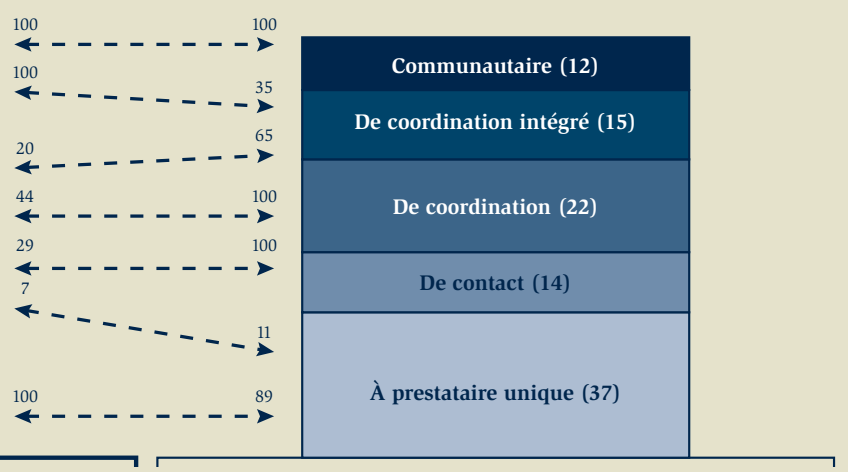

Modèles d'organisation de SSP

${ }^{a}$ Les pourcentages se lisent de la manière suivante : 100 des CLSC (bloc de gauche) appartiennent au modèle communautaire (bloc de droite) et ils constituent $100 \%$ de cette catégorie.

${ }^{\mathrm{b}}$ CLSC : Centres locaux de services communautaires

' GMF : Groupes de médecine familiale

FIGURE 2

Diagramme d'affectation des répondants de l'enquête à un groupe de maladies chroniques

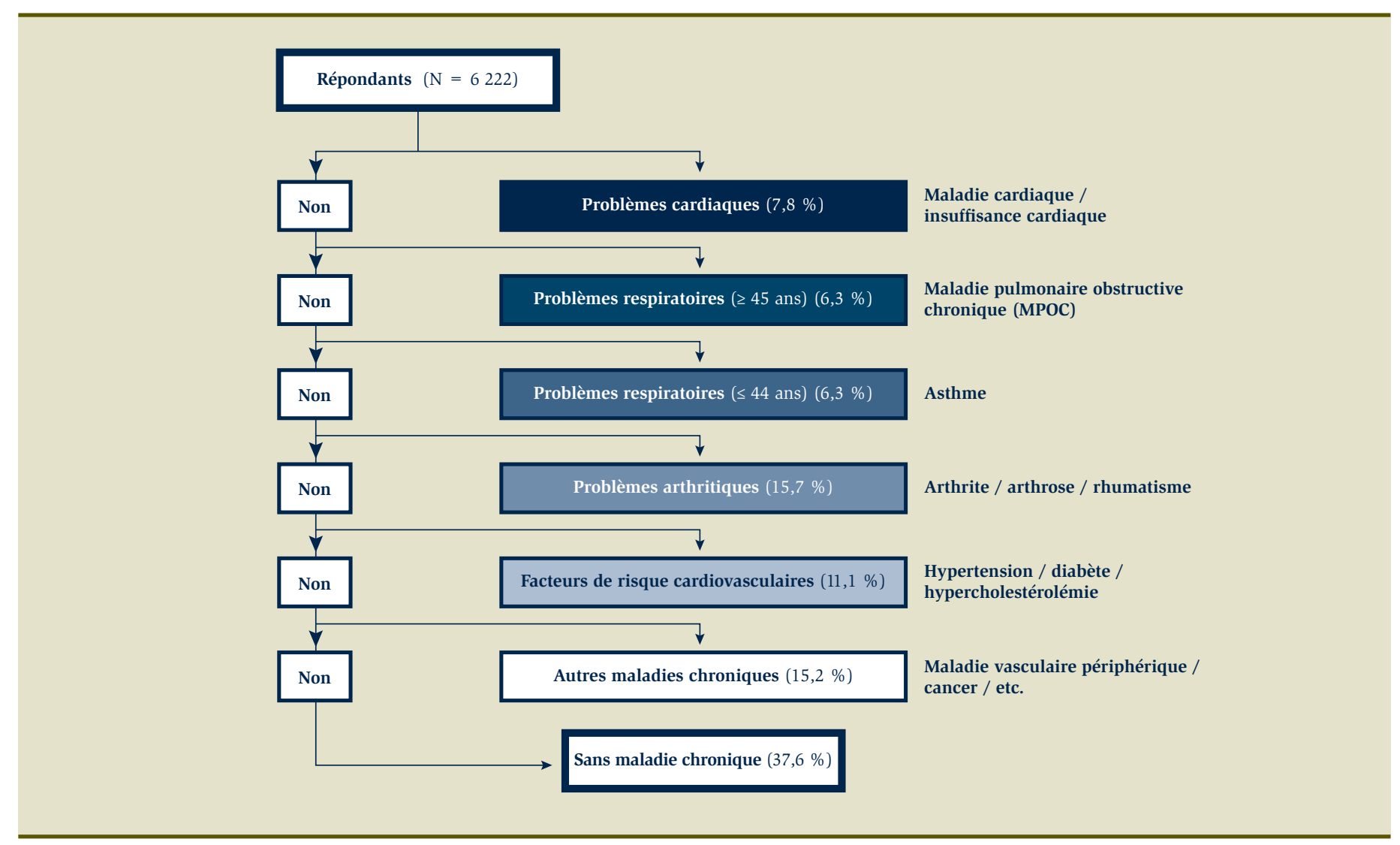


TABLEAU 3

Caractéristiques des répondants de l'enquête populationnelle ( $\mathrm{N}=6$ 222) selon le groupe de maladies chroniques, Québec, 2003-2005

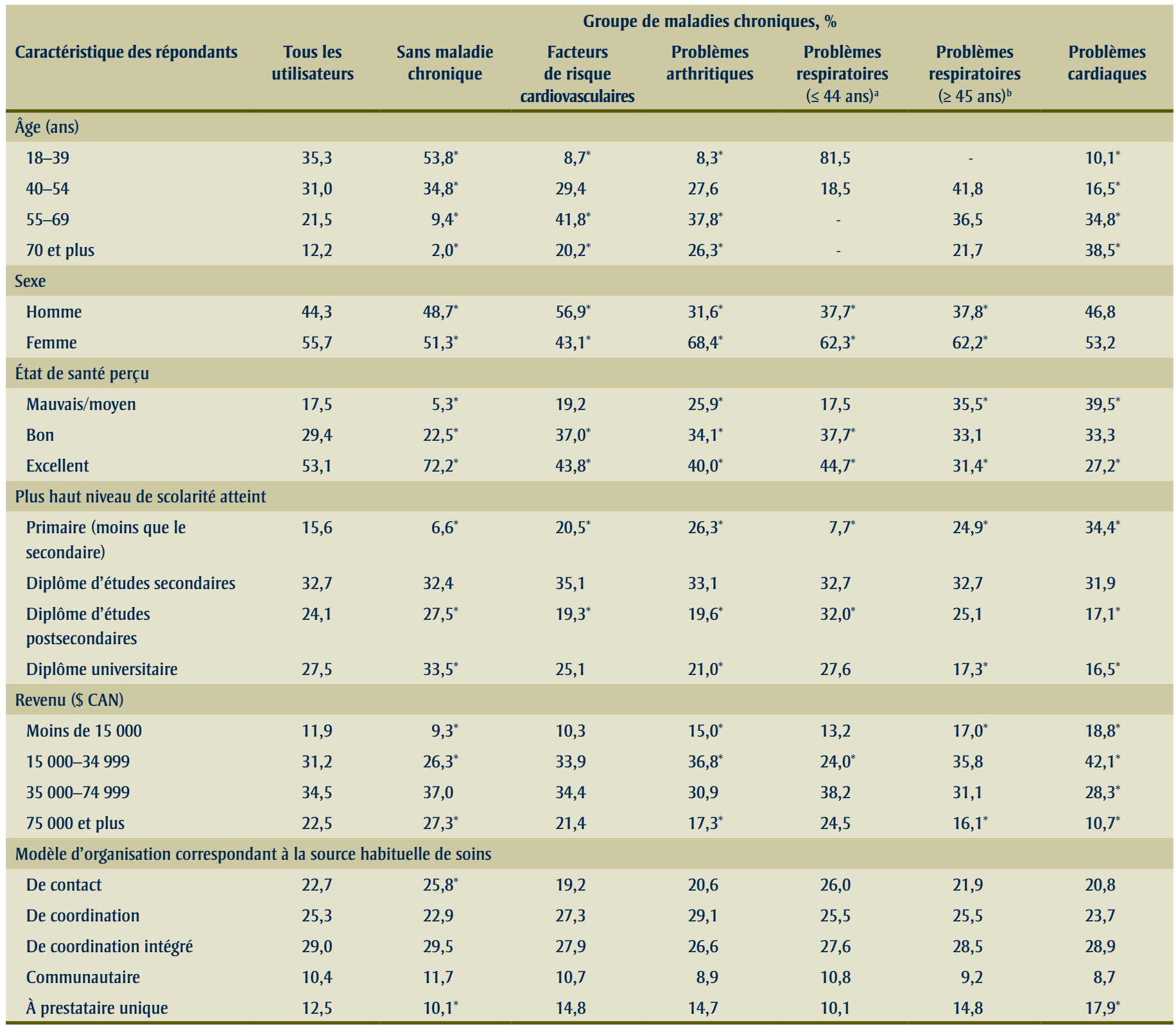

Abréviations : \$CAN, dollars canadiens; N, taille totale de l'échantillon; p, signification statistique.

${ }^{a}$ La principale maladie dans ce groupe d'âge ( $\leq 44$ ans) est probablement l'asthme.

${ }^{\mathrm{b}}$ La principale maladie dans ce groupe d'âge ( $\geq 45$ ans) est probablement la maladie pulmonaire obstructive chronique (MPOC).

${ }^{*} p \leq 0,05$; le groupe comprenant tous les utilisateurs sert de référence.

ne comprenne aucun patient atteint d'une maladie chronique, les personnes souffrant d'une maladie chronique autre que celles visées par l'étude $(15,2 \%)$ ont été exclues des analyses. Les facteurs de risque cardiovasculaires comprennent le diabète, l'hypertension et l'hypercholestérolémie. Dans le cas des autres maladies chroniques, il n’était en général pas possible de faire des distinctions plus précises au sein de chaque catégorie.

Dans le but d'évaluer l'association entre les différents modèles d'organisation et l'expérience de soins des malades chroniques, nous avons effectué des régressions logistiques stratifiées des trois variables dichotomiques d'intérêt (accès, processus, résultats) pour chaque groupe de maladies chroniques. Pour toutes les analyses, nous avons utilisé l'âge, le sexe, le revenu et le niveau d'instruction comme covariables. 


\section{Résultats}

\section{Caractéristiques individuelles et affiliation des répondants}

Le tableau 3 présente les caractéristiques des répondants, notamment leur affiliation à un modèle communautaire ou professionnel de soins. Chaque groupe de maladie, y compris le groupe de personnes sans maladie chronique, est comparé au groupe de référence formé de l'ensemble des utilisateurs. Si on les compare au groupe de référence, les personnes $\mathrm{du}$ groupe sans maladie chronique sont en général plus jeunes, en meilleure santé, de sexe masculin et plus instruites. Par ailleurs, le groupe des facteurs de risque cardiovasculaires comprend davantage de personnes plus âgées, de sexe masculin et moins instruites que le groupe de référence. Les groupes des problèmes arthritiques, respiratoires (personnes de 45 ans et plus) et cardiaques partagent certaines caractéristiques : ils sont formés de personnes plus âgées, en moins bonne santé, moins instruites et ayant un revenu moins élevé que celles du groupe de référence. Les groupes des problèmes arthritiques et respiratoires comprennent un pourcentage plus élevé de femmes. Les personnes du groupe des problèmes respiratoires (personnes de 44 ans et moins) sont plus jeunes et, de façon générale, ont un niveau d'instruction et des revenus plus élevés.

On observe peu de variation entre les groupes de maladies quant à l'affiliation aux divers modèles d'organisation de SSP (tableau 3). On note toutefois une exception : si on les compare à l'ensemble des utilisateurs, les répondants du groupe sans maladie chronique se concentrent davantage dans le modèle de contact et se retrouvent moins souvent dans le modèle à prestataire unique. Ce dernier attire plus que sa part de personnes atteintes de maladies cardiaques, et moins de personnes avec problèmes respiratoires (de 44 ans et moins). Mis à part ces différences, le pourcentage de personnes affiliées aux modèles d'organisation dans les différents groupes des maladies chroniques est semblable à celui qu'on retrouve pour l'ensemble des utilisateurs. Toutefois, le pourcentage d'utilisateurs de services qui identifient l'un des modèles comme étant leur source habituelle de soins varie considérablement; il s'élève à 10,4 \% pour le modèle communautaire et atteint $29,0 \%$ pour le modèle de coordination intégré.

Lorsqu'on passe du groupe sans maladie chronique aux groupes des maladies cardiaques (tableau 3), l'état de santé perçu a tendance à se détériorer, ce qui semble refléter un gradient croissant de gravité de la maladie.

Une analyse plus approfondie des états comorbides associés à ces maladies chroniques permet de confirmer ce degré croissant de gravité, puisque le nombre d'états comorbides associés à la maladie chronique principale augmente de façon constante lorsqu'on passe du groupe sans maladie chronique au groupe des maladies cardiaques (tableau 4).

\section{L'expérience de soins selon la maladie}

L'expérience de soins varie selon les différentes maladies (tableau 5). L'accessibilité de premier contact présente le plus faible pourcentage de personnes ayant un score de 7,5 et plus, ainsi que le moins de variation selon les maladies. Elle est légèrement inférieure chez les personnes du groupe sans maladie chronique, bien qu'il n'y ait aucune différence statistiquement significative entre chaque catégorie de maladies chroniques et l'ensemble des utilisateurs.

Le processus de soins affiche un pourcentage plus élevé de personnes ayant un score de 7,5 et plus ainsi qu'une plus grande variation selon les maladies que l'accessibilité de premier contact. Le groupe des problèmes respiratoires (personnes de 44 ans et moins) présente le pourcentage le moins élevé de personnes ayant un score de 7,5 et plus, un résultat qui est même inférieur à celui du groupe sans maladie chronique, tandis que le groupe des facteurs de risque cardiovasculaires obtient le pourcentage le plus élevé. Les résultats des soins affichent le même profil que le processus de soins : le pourcentage de personnes ayant un score de 7,5 et plus varie; il est beaucoup plus faible chez les personnes de 44 ans et moins atteintes de maladies respiratoires et il est plus élevé chez les personnes qui présentent des facteurs de risque cardiovasculaires.

\section{Expérience de soins liée aux modèles d'organisation selon les différentes maladies}

Le tableau 6 présente des rapports de cote (RC) avec des intervalles de confiance (IC) à $95 \%$ pour les relations entre les modèles d'organisation et l'expérience de soins dans les différents groupes de maladies chroniques, le modèle professionnel de contact servant de référence. Les données ont été ajustées en fonction des caractéristiques des répondants, sauf dans le cas de l'état de santé perçu, qui était fortement corrélé avec les maladies chroniques visées par les analyses.

L'accessibilité de premier contact est meilleure pour les modèles de contact et de coordination intégré $(\mathrm{RC}>1$; limite inférieure de l'IC $\geq 1$ ) et bien pire pour le modèle à prestataire unique $(\mathrm{RC}<1$; limite supérieure de l'IC < 1) dans tous les groupes de maladies chroniques et le groupe sans maladie chronique. Le modèle communautaire figure également parmi les modèles les plus accessibles pour le groupe des problèmes arthritiques et les deux groupes d'âge avec problèmes respiratoires.

Les rapports de cote pour le processus de soins varient également selon le modèle d'organisation et la maladie. Le modèle de contact offre en général un processus de soins moins favorable que les autres modèles dans tous les groupes de maladies ainsi que dans le groupe sans maladie chronique, sauf en ce qui concerne le groupe des facteurs de risque cardiovasculaires et le groupe des maladies respiratoires (personnes de 44 ans et moins). Pour ces deux groupes, tous les modèles d'organisation autres que le modèle à prestataire unique affichent une expérience de soins moins favorable.

Les données relatives aux résultats des soins présentent le même profil que celles du processus de soins. Celles du groupe sans maladie chronique suivent de façon plus étroite le profil observé pour le processus de soins. Les autres données ressemblent à celles obtenues pour le processus de soins, mais la plupart d'entre elles ne sont pas statistiquement significatives. 
TABLEAU 4

Répondants de l'enquête populationnelle $(\mathbf{N}=6$ 222) qui présentent des états comorbides associés à leur groupe de maladies chroniques

\begin{tabular}{|c|c|c|c|c|c|}
\hline \multirow[b]{2}{*}{ Groupe de maladies chroniques } & \multicolumn{5}{|c|}{ États comorbides en \% } \\
\hline & $\begin{array}{l}\text { Problèmes } \\
\text { cardiaques }\end{array}$ & $\begin{array}{l}\text { Problèmes } \\
\text { respiratoires }\end{array}$ & $\begin{array}{l}\text { Problèmes } \\
\text { arthritiques }\end{array}$ & $\begin{array}{c}\text { Facteurs de risque } \\
\text { cardiovasculaires }\end{array}$ & $\begin{array}{c}\text { Autres problèmes } \\
\text { de santé }\end{array}$ \\
\hline Problèmes cardiaques & 100,0 & 24,3 & 45,9 & 65,4 & 74,9 \\
\hline Problèmes respiratoires ( $\geq 45$ ans) ${ }^{a}$ & - & 100,0 & 50,0 & 38,9 & 67,2 \\
\hline Problèmes respiratoires ( $\leq 44$ ans) ${ }^{b}$ & - & 100,0 & 9,9 & 3,6 & 38,2 \\
\hline Problèmes arthritiques & - & - & 100,0 & 39,1 & 51,1 \\
\hline Facteurs de risque cardiovasculaires & - & - & - & 100,0 & 39,9 \\
\hline Autres problèmes de santé & - & - & - & - & 100,0 \\
\hline Sans maladie chronique & - & - & - & - & - \\
\hline
\end{tabular}

Abréviation : N, taille totale de l'échantillon.

a La principale maladie dans ce groupe d'âge ( $\geq 45$ ans) est probablement la maladie pulmonaire obstructive chronique (MPOC).

${ }^{\mathrm{b}}$ La principale maladie dans ce groupe d'âge ( $\leq 44$ ans) est probablement l'asthme.

TABLEAU 5

Répondants de l'enquête populationnelle ( $N=6222)$ ayant rapporté une meilleure expérience de soins ${ }^{\mathrm{a}}$ selon le groupe de maladies chroniques

\begin{tabular}{|c|c|c|c|c|c|c|c|}
\hline \multirow[b]{2}{*}{ Expérience de soins } & \multicolumn{7}{|c|}{ Groupe de maladies chroniques, en \% } \\
\hline & $\begin{array}{c}\text { Tous les } \\
\text { utilisateurs }\end{array}$ & $\begin{array}{l}\text { Sans maladie } \\
\text { chronique }\end{array}$ & $\begin{array}{c}\text { Facteurs } \\
\text { de risque } \\
\text { cardiovasculaires }\end{array}$ & $\begin{array}{l}\text { Problèmes } \\
\text { arthritiques }\end{array}$ & $\begin{array}{l}\text { Problèmes } \\
\text { respiratoires } \\
(\leq 44 \text { ans })^{b}\end{array}$ & $\begin{array}{l}\text { Problèmes } \\
\text { respiratoires } \\
(\geq 45 \text { ans })^{c}\end{array}$ & $\begin{array}{l}\text { Problèmes } \\
\text { cardiaques }\end{array}$ \\
\hline Accessibilité de premier contact & 29,7 & 28,4 & 31,7 & 32,1 & 29,1 & 30,7 & 30,3 \\
\hline Processus de soins & 61,4 & $54,7^{*}$ & $79,1^{*}$ & $69,0^{*}$ & $48,8^{*}$ & $71,5^{*}$ & $69,5^{*}$ \\
\hline Résultats des soins & 56,8 & $52,4^{*}$ & $73,4^{*}$ & $62,5^{*}$ & $42,5^{*}$ & $63,9^{*}$ & $62,9^{*}$ \\
\hline
\end{tabular}

Abréviations : $\mathrm{N}$, taille de l'échantillon; $p$, signification statistique.

a Score de 7,5 sur 10 sur une échelle de 0 à 10 de scores dichotomisés.

${ }^{\text {b }}$ La principale maladie dans ce groupe d'âge ( $\leq 44$ ans) est probablement l'asthme.

' La principale maladie dans ce groupe d'âge ( $\geq 45$ ans) est probablement la maladie pulmonaire obstructive chronique (MPOC).

${ }^{*} p \leq 0,05$; le groupe comprenant l'ensemble des utilisateurs sert de référence

\section{Discussion}

Dans le cadre de cette étude, nous avons mis en lumière l'étendue des expériences de soins selon les maladies chroniques. Nous avons également exploré la relation entre les modèles d'organisation de SSP et l'expérience de soins afin de déterminer dans quelle mesure cette relation varie selon différents types de maladies chroniques.

Deux constatations importantes ressortent de notre étude. Premièrement, l'accessibilité des soins est relativement faible pour tous les groupes de maladies chroniques ainsi que pour le groupe sans maladie chronique, et elle présente peu de variation selon les maladies. Les indices du processus de soins et des résultats des soins sont beaucoup plus élevés, et ce, pour tous les groupes. De plus, ils varient selon les maladies, les plus élevés se trouvant dans le groupe avec facteurs de risque cardiovasculaires et les plus faibles, dans celui avec maladies respiratoires (personnes de 44 ans et moins).

Deuxièmement, l'accessibilité de premier contact pour toutes les maladies chroniques est plus élevée dans les modèles de contact et de coordination intégré que dans les autres modèles. Inversement, l'accessibilité de premier contact est souvent plus faible chez les patients dont la source régulière de soins correspond aux modèles d'organisation de SSP à prestataire unique ou de coordination.

À l'opposé, pour le processus de soins et, dans une moindre mesure, les résultats des soins, le modèle à prestataire unique est associé à de meilleures performances que le modèle de contact pour tous les groupes (avec ou sans maladie chronique). Les patients qui présentent des facteurs de risque cardiovasculaires et des maladies respiratoires (âgés de 44 ans et moins) rapportent un moins bon processus de soins pour tous les modèles, à l'exception de celui à prestataire unique, tandis que pour le groupe sans maladie chronique et les groupes des maladies arthritiques, respiratoires (personnes de 45 ans et plus) et cardiaques, tous les modèles sont supérieurs au modèle de contact. Le modèle communautaire est supérieur chez les patients plus âgés (45 ans et plus) atteints de maladies respiratoires, tandis que le modèle de coordination est supérieur chez les personnes atteintes de maladies cardiaques (tableau 6).

Ces constatations sur l'accessibilité méritent d'être expliquées de façon plus détaillée. Premièrement, dans notre étude, le pourcentage de personnes ayant un score élevé 


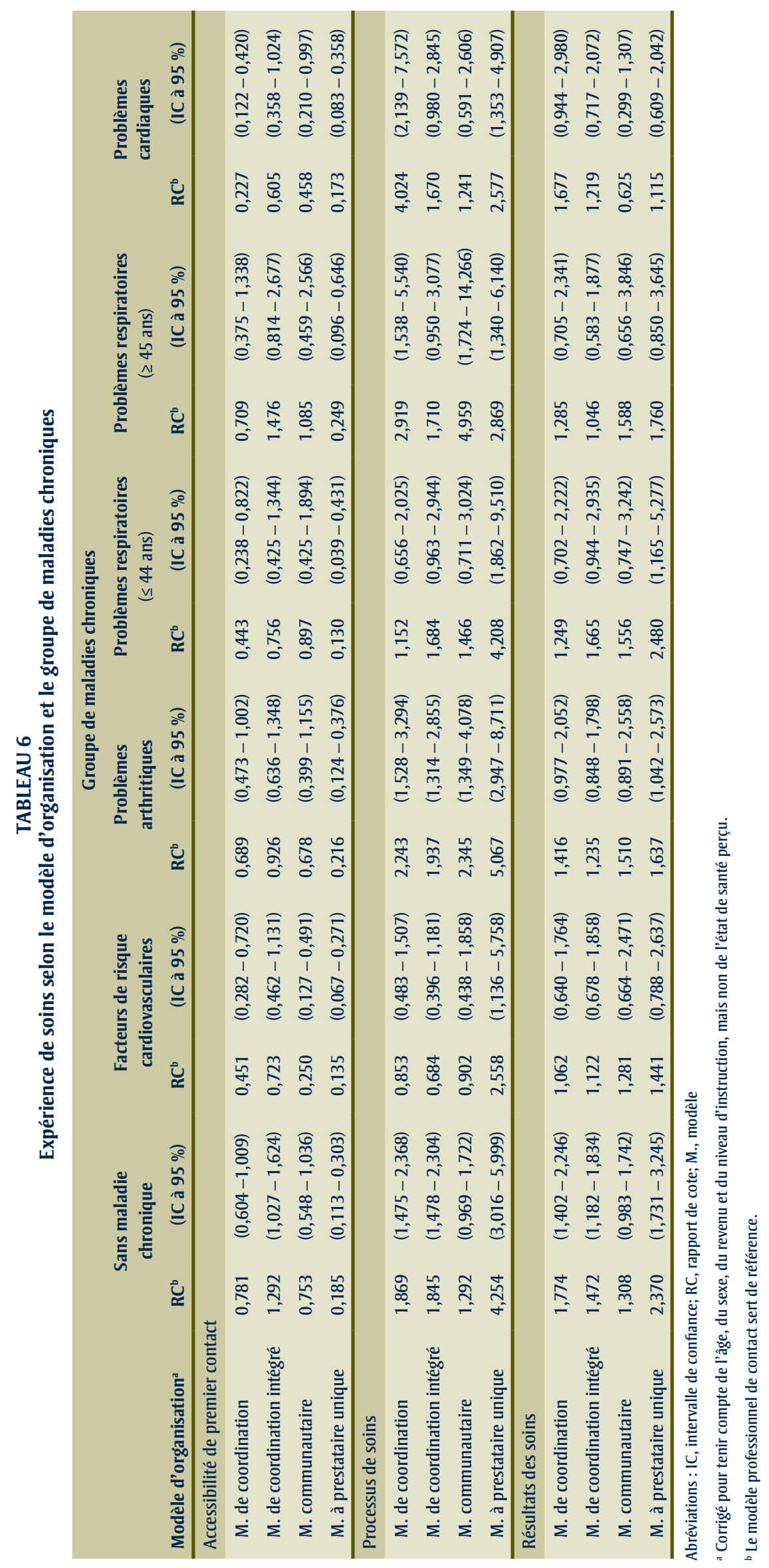

( $\geq 7,5$ sur 10) pour l'accessibilité de premier contact à des SSP est plutôt faible (de 28,4 à $32,1 \%$ ), peu importe leur état de santé, et ce pourcentage est beaucoup plus faible que celui des autres caractéristiques de l'expérience de soins (tableau 5). D'autres études ont également fait état de graves problèmes d'accessibilité aux services de $\mathrm{SSP}^{28-30}$. Bien que la variation entre les modèles soit minime, les analyses de régression logistique font ressortir deux résultats intéressants et contradictoires : le modèle à prestataire unique présente la moins grande accessibilité de premier contact, tandis que le modèle de contact présente la plus grande accessibilité peu importe l'état de santé. Puisqu'un pourcentage plus élevé de patients affiliés au modèle à prestataire unique que de patients affiliés au modèle de contact a un médecin régulier (94\% contre $64 \%$ ), il semble que le fait d'avoir un médecin régulier ne figure pas parmi les principaux facteurs favorisant l'accessibilité (figure 3$)^{29,31-33}$. En ce qui concerne cette dimension de l'expérience de soins, le fait d'avoir un médecin régulier ne semble donc pas être le seul facteur important pouvant expliquer la relation entre l'accessibilité de premier contact et les modèles d'organisation de SSP; certains attributs intrinsèques de ces divers modèles, comme la pratique de groupe, semblent également importants. Cela découle du fait que l'accès aux soins de santé est conceptualisé dans la présente étude comme étant l'accès du patient à un médecin généraliste en particulier, ainsi qu'à d'autres médecins si son médecin de famille est absent. De toute évidence, les médecins qui exercent en solo ne répondent pas à cette vision élargie de l'accessibilité de premier contact. Inversement, le modèle de contact possède des caractéristiques intrinsèques qui favorisent l'accessibilité de premier contact (tableau 2).

Si on les compare à l'accessibilité de premier contact, le processus de soins et, dans une moindre mesure, les résultats des soins affichent des pourcentages beaucoup plus élevés de personnes ayant des scores élevés pour toutes les maladies. On constate également une plus grande variation entre les maladies, le groupe des maladies respiratoires (personnes de 44 ans et moins) présentant le pourcentage le plus faible et le groupe 
FIGURE 3

Pourcentage de répondants de l'enquête ( $\mathrm{N}=6$ 222) ayant un médecin régulier selon le modèle d'organisation correspondant au type de pratique de ce médecin

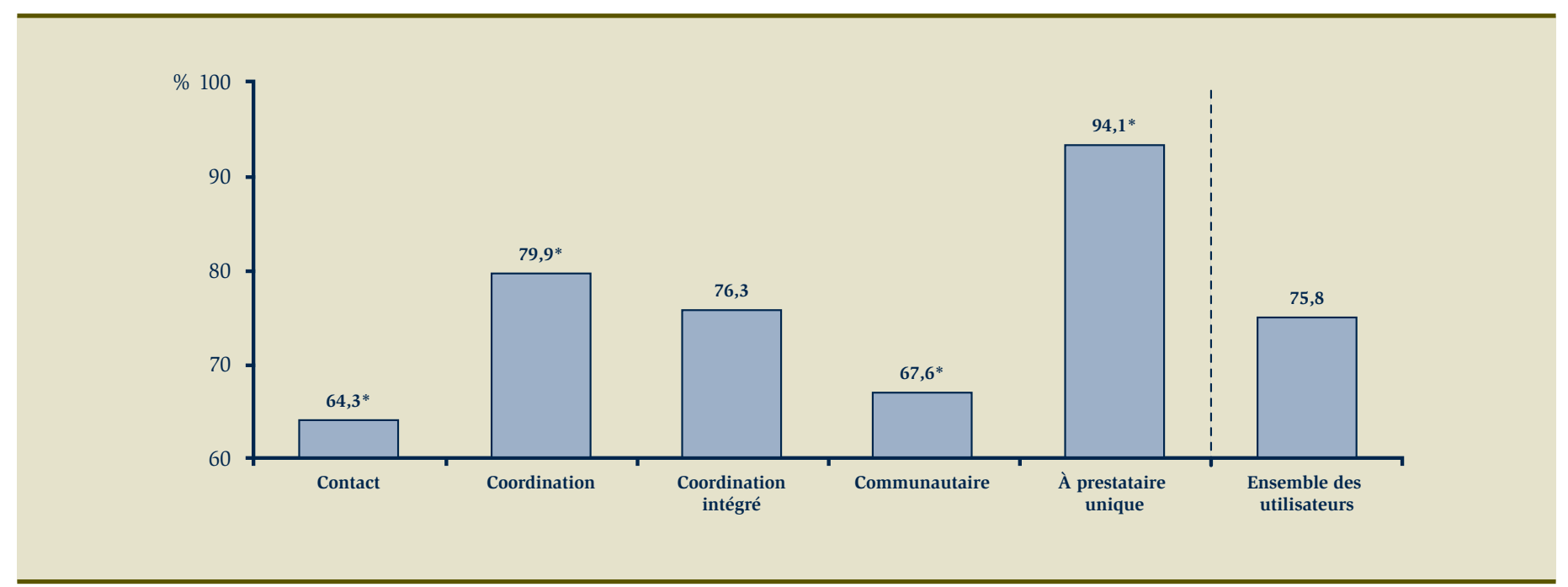

${ }^{*} p \leq 0,05$; le groupe comprenant tous les utilisateurs sert de référence.

FIGURE 4

Pourcentage de répondants de l'enquête $(\mathrm{N}=6$ 222) ayant un médecin régulier selon le groupe de maladies chroniques

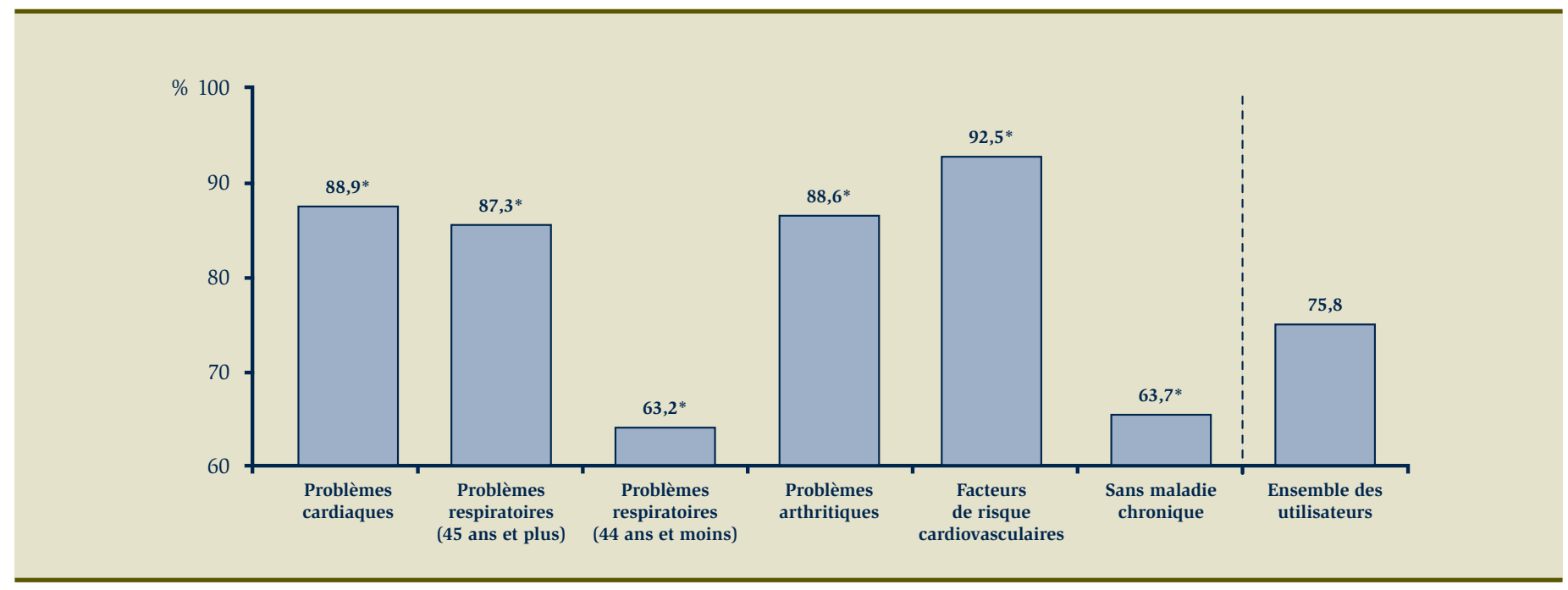

${ }^{*} p \leq 0,05$; le groupe comprenant tous les utilisateurs sert de référence.

des facteurs de risque cardiovasculaires, le plus élevé (tableau 5). Ces différences pourraient refléter le fait que dans le groupe des maladies respiratoires (personnes de 44 ans et moins), moins de patients ont un médecin régulier (63\%) que dans le groupe des facteurs de risque cardiovasculaires (93\%) (figure 4). Les patients du groupe des maladies respiratoires (personnes de 44 ans et moins) sont également plus jeunes que ceux du groupe des facteurs de risque cardiovasculaires (tableau 3). Des études ont révélé que les patients plus âgés et les patients ayant un médecin régulier sont plus nombreux à rapporter avoir vécu une expérience de soins favorable $e^{29,31,32}$. Si on le compare à l'accessibilité, le processus de soins obtient un résultat beaucoup plus élevé pour les patients de tous les groupes de maladies qui sont affiliés au modèle de SSP à prestataire unique (tableau 6).

Les deux modèles de coordination et le modèle communautaire affichent aussi un meilleur processus de soins et de meilleurs résultats de soins que le modèle de contact pour le groupe sans maladie chronique et les groupes des maladies arthritiques et respiratoires (personnes de 45 ans et plus) (tableau 6). Cela indique qu'au moins pour ces trois groupes, les modèles d'organisation influent sur ces aspects de l'expérience de soins, bien qu'une partie de cette influence puisse être attribuable à l'âge des patients et au fait d'avoir un médecin régulier. Toutefois, cette explication ne convient pas 
pour le groupe de patients plus jeunes sans maladie chronique, dans lequel le pourcentage de personnes ayant un médecin régulier est plus faible. Finalement, ces deux facteurs - l'âge et le fait d'avoir un médecin régulier - expliquent probablement l'absence de relation, chez le groupe de patients plus jeunes atteints d'une maladie respiratoire, entre les modèles et l'expérience de soins, sauf en ce qui concerne le modèle à prestataire unique. Ces constatations semblent indiquer une interaction possible entre l'âge et le fait d'avoir un médecin régulier, une interaction que nous n'avons pas explorée plus avant. Le profil divergent que nous avons observé pour le groupe des facteurs de risque cardiovasculaires est difficile à expliquer. En fait, on pourrait s'attendre à ce que l'âge et le fait d'avoir un médecin régulier contribuent à une meilleure expérience de soins que celle vécue dans le modèle de contact. L'absence de différence entre les modèles reflète probablement le fait que les patients qui présentent des facteurs de risque cardiovasculaires sont moins sensibles aux caractéristiques différentielles des modèles de SSP et voient leurs besoins satisfaits de façon équivalente par les divers modèles d'organisation. Il est également plausible que ces patients présentent moins de symptômes et qu'ils aient ainsi moins besoin de soins médicaux soutenus. Cette hypothèse devra être vérifiée dans le cadre d'autres analyses.

De façon générale, le modèle professionnel de coordination intégré et le modèle communautaire ressortent comme étant ceux qui, généralement, performent le mieux, qu'il s'agisse de l'expérience de soins, de l'accessibilité, du processus de soins ou des résultats des soins, et ce, pour la plupart des groupes. Il convient de noter que ces deux modèles montrent un processus de soins plus favorable pour les groupes de maladies plus graves, comme les maladies cardiaques, respiratoires (personnes de 45 ans et plus) et arthritiques. Comme nous l'avons déjà mentionné, ces maladies plus graves sont également accompagnées d'un plus grand nombre d'états comorbides; une approche globale et intégrée est donc nécessaire afin de répondre aux divers besoins. C'est pourquoi le modèle de coordination intégré et le modèle communautaire sont particulièrement adaptés pour relever le défi croissant que pose la prise en charge des maladies chroniques.

Bien que les données relatives au processus de soins affichent un profil semblable à celles des résultats des soins, la plupart de ces dernières ne sont pas statistiquement significatives. Cela pourrait s'expliquer par un manque de puissance statistique, mais aussi par le manque de spécificité de nos indicateurs de résultats, qui sont en grande partie liés à la prévention. La relation ténue entre le processus de soins et les résultats des soins est souvent observée dans les études portant sur l'expérience de soins et la continuité ${ }^{34}$.

Finalement, nos conclusions doivent aussi être interprétées à la lumière de l'importance relative des cinq modèles d'organisation de SSP présentés. Comme on peut le voir dans le tableau 3 , les trois modèles professionnels que sont les modèles de contact, de coordination et de coordination intégré partagent plus de $75 \%$ de l'ensemble de l'utilisation, tandis que les modèles communautaire et à prestataire unique représentent à peine plus de $10 \%$ chacun. De plus, le modèle à prestataire unique est un modèle d'organisation de SSP en déclin, tandis que le modèle communautaire n'a pas démontré sa capacité à se développer au-delà de son niveau actuel. Par conséquent, les améliorations notables à notre système de soins de santé devraient provenir des trois modèles professionnels les plus utilisés, ce qui pourrait se faire soit en amenant ces derniers à ressembler davantage au modèle le plus performant, que nous avons identifié dans la présente étude comme étant le modèle de coordination intégré, soit en établissant des réseaux au sein desquels chacun des modèles remplirait des fonctions particulières et complémentaires d'une manière coordonnée et intégrée.

\section{Points forts et limites}

Notre étude comporte certaines limites. D'abord, en raison de la nature transversale de l'étude, il est difficile d'établir des liens de causalité entre les modèles de soins et l'expérience de soins rapportée au cours des deux années précédentes. De plus, le biais de rappel peut limiter l'exactitude et la fiabilité des données recueillies sur l'expérience de soins.
L'autodéclaration des maladies chroniques constitue une autre limite. Même si la formulation de la question faisait référence à la validation du diagnostic par un médecin (" Est ce qu’un médecin vous a déjà dit que vous faites du diabète? »), la réponse est toujours limitée par l'interprétation subjective du répondant et par sa capacité à rapporter avec exactitude des renseignements de nature médicale. De même, il a été impossible d'obtenir une plus grande spécificité diagnostique. Par exemple, nous avons divisé le groupe des maladies respiratoires en deux catégories d'âge, en tenant pour acquis que les personnes de 44 ans et moins déclaraient principalement être atteintes d'asthme, tandis que celles de 45 ans et plus souffraient principalement de MPOC, mais nous n'avons pas été en mesure de valider ces hypothèses.

En outre, le fait de classer les maladies dans des catégories mutuellement exclusives a pour effet d'ajouter davantage d'états comorbides dans les catégories apparaissant en premier dans l'échelle. Cette façon de faire accroît par le fait même l'hétérogénéité de ces catégories. Cependant, compte tenu de la corrélation entre l'état de santé perçu et les catégories de maladies chroniques, nous considérons que l'ordre d'inclusion des catégories de maladies représente avec justesse le degré décroissant de gravité. Toutefois, puisqu'il s'agit d'une mesure de prévalence d'un diagnostic et non d'une véritable mesure de l'état de santé, nous ne pouvons prétendre avoir pleinement pris en compte la gravité de la maladie et son effet potentiel sur l'expérience de soins. Finalement, l'autosélection des patients dans les différents modèles d'organisation de soins primaires ne peut être complètement écartée.

Notre étude comporte aussi certains points forts. En nous appuyant sur un vaste échantillon de l'ensemble de la population des deux régions les plus peuplées du Québec et en envoyant un questionnaire à toutes les organisations de SSP de ces deux régions, nous avons pu jumeler $89 \%$ des répondants à l'une ou l'autre des 473 organisations de SSP étudiées. 


\section{Conclusion}

Les résultats de notre étude révèlent que les divers modèles d'organisation de SSP affichent des performances différentes selon les maladies chroniques étudiées. L'accessibilité des soins obtient le résultat le plus faible pour tous les groupes de maladies chroniques et affiche peu de variation selon les maladies. Les modèles de contact et de coordination intégré sont les plus accessibles, tandis que le modèle à prestataire unique est le moins accessible. Les indices du processus de soins et des résultats des soins sont beaucoup plus élevés que l'indice de l'accessibilité, et ce, pour tous les groupes. De plus, ils varient selon les maladies, les plus élevés se trouvant dans le groupe des facteurs de risque cardiovasculaires, et les plus faibles, dans le groupe de patients plus jeunes (44 ans et moins) atteints de maladies respiratoires. Le modèle de contact semble être le meilleur sur le plan de l'accessibilité, tandis que le modèle à prestataire unique serait le meilleur en ce qui a trait au processus de soins. Ces deux modèles comportent néanmoins d'importantes limites lorsqu'on s'attarde aux autres aspects de l'expérience de soins. Dans le cas des maladies chroniques plus graves, le modèle de coordination intégré et le modèle communautaire offrent en général un meilleur processus de soins et, par conséquent, risquent davantage de satisfaire aux conditions essentielles à la mise en œuvre fructueuse du modèle de soins aux malades chroniques. Le modèle de coordination intégré ressort tout particulièrement comme étant le modèle le plus complet; il obtient des scores plus élevés pour l'accessibilité et le processus de soins pour presque toutes les maladies chroniques et il présente un degré élevé d'utilisation des services. En ce sens, ce modèle recèle probablement le plus grand potentiel pour instaurer des changements importants dans notre système de soins de santé.

\section{Remerciements}

Les données présentées dans le présent article sont issues d'un projet de recherche financé par les Instituts de recherche en santé du Canada (IRSC), la Fondation canadienne de la recherche sur les services de santé (FCRSS) et le Fonds de la recherche en santé du
Québec (FRSQ). Ce projet a également reçu un appui financier de l'Agence de la santé et des services sociaux de Montréal et de l'Agence de la santé et des services sociaux de la Montérégie, de l'Institut national de santé publique du Québec (INSPQ), du Groupe de recherche sur l'équité d'accès et l'organisation des services de santé de 1re ligne (GRÉAS 1) et du Groupe interuniversitaire de recherche sur les urgences (GIRU).

Les auteurs déclarent n'avoir aucun conflit d'intérêts.

Les auteurs aimeraient remercier Alexandre Prud'homme, Odette Lemoine et Brigitte Simard pour leur contribution à l'analyse des données, ainsi que Sylvie Gauthier et Isabelle Rioux, qui ont révisé le texte et contribué à la rédaction du manuscrit.

\section{Références}

1. Broemeling AM, Watson DE, Prebtani F. Population patterns of chronic health conditions, co-morbidity and healthcare use in Canada: implications for policy and practice. Healthc Q. 2008;11(3):70-6.

2. Wagner EH. Meeting the needs of chronically ill people. BMJ. 2001;323(7319):945-6.

3. Epping-Jordan JE, Pruitt SD, Bengoa R, Wagner EH. Improving the quality of health care for chronic conditions. Qual Saf Health Care. 2004;13(4):299-305.

4. Starfield B, Lemke KW, Bernhardt T, Foldes SS, Forrest CB, Weiner JP. Comorbidity: implications for the importance of primary care in 'case' management. Ann Fam Med. 2003;1(1):8-14.

5. Starfield B, Lemke KW, Herbert R, Pavlovich WD, Anderson G. Comorbidity and the use of primary care and specialist care in the elderly. Ann Fam Med. 2005;3(2):215-22.

6. Grumbach K. Chronic illness, comorbidities, and the need for medical generalism. Ann Fam Med. 2003;1(1):4-7.

7. Broemeling AM, Watson DE, Black C. Chronic conditions and co-morbidity among residents of British Columbia. Vancouver (BC): Centre for Health Services and Policy Research, University of British Columbia; 2005.
8. Rothman AA, Wagner EH. Chronic illness management: what is the role of primary care? Ann Intern Med. 2003;138(3):256-61.

9. Davis K, Schoenbaum SC, Audet AM. A 2020 vision of patient-centered primary care. J Gen Intern Med. 2005;20(10):953-7.

10. Starfield B, Shi L. The medical home, access to care, and insurance: a review of evidence. Pediatrics. 2004;113(5 Suppl):1493-8.

11. O'Connor PJ, Sperl-Hillen JM, Pronk NP, Murray T. Primary care clinic-based chronic disease care: Features of successful programs. Dis Manag Health Out. 2001;9(12):691-8.

12. Bodenheimer T, Wagner EH, Grumbach K. Improving primary care for patients with chronic illness: the chronic care model, Part 2. JAMA. 2002;288(15):1909-14.

13. Barr VJ, Robinson S, Marin-Link B, Underhill L, Dotts A, Ravensdale D, et coll. The expanded Chronic Care Model: an integration of concepts and strategies from population health promotion and the Chronic Care Model. Hosp Q. 2003;7(1):73-82.

14. Rundall TG, Shortell SM, Wang MC, Casalino L, Bodenheimer T, Gillies RR, et coll. As good as it gets? Chronic care management in nine leading US physician organizations. BMJ. 2002;325(7370):958-61.

15. Levesque JF, Feldman D, Dufresne C, Bergeron P, Pinard B, Gagné V. Barrières et éléments facilitant l'implantation de modèles intégrés de prévention et de gestion des maladies chroniques. Prat Organ Soins. 2009;40(4):251-65.

16. Friedberg MW, Coltin KL, Safran DG, Dresser M, Zaslavsky AM, Schneider EC. Associations between structural capabilities of primary care practices and performance on selected quality measures. Ann Intern Med. 2009;151(7):456-63.

17. Shortell SM, Marsteller JA, Lin M, Pearson ML, Wu SY, Mendel P, et coll. The role of perceived team effectiveness in improving chronic illness care. Med Care. 2004;42(11):1040 8. 
18. Russell GM, Dahrouge S, Hogg W, Geneau R, Muldoon L, Tuna M. Managing chronic disease in Ontario primary care: the impact of organizational factors. Ann Fam Med. 2009;7(4):309-18.

19. Glazier RH, Klein-Geltink J, Kopp A, Sibley LM. Capitation and enhanced fee-for-service models for primary care reform: a population-based evaluation. CMAJ. 2009;180(11):E72-E81.

20. Tsai AC, Morton SC, Mangione CM, Keeler EB. A meta-analysis of interventions to improve care for chronic illnesses. Am J Manag Care. 2005;11(8):478-88.

21. Cretin S, Shortell SM, Keeler EB. An evaluation of collaborative interventions to improve chronic illness care: Framework and study design. Eval Rev. 2004;28(1):28-51.

22. Levesque JF, Pineault R, Simard B, Roberge D, Hamel M, Kapetanakis C, et coll. L'expérience de soins de la population : portrait des variations intra-régionales à Montréal et en Montérégie [Internet]. Montréal (Québec) : Institut national de santé publique du Québec; 2007 [consultation le 8 novembre 2010]. PDF téléchargeable à partir du lien : http://www.inspq.qc.ca /pdf/publications/627-ExperienceDeSoinsDeLaPopulation.pdf. Publié conjointement par la Direction de santé publique de l'Agence de la santé et des services sociaux de Montréal.

23. Pineault R, Levesque JF, Roberge D, Hamel M, Lamarche P, Haggerty J. L'accessibilité et la continuité des services de santé : une étude sur la première ligne au Québec : rapport de recherche soumis aux Instituts de recherche en santé du Canada (IRSC) et à la Fondation canadienne de la recherche sur les services de santé (FCRSS). [Internet]. Montréal (Québec) : Direction de santé publique de l'Agence de la santé et des services sociaux de Montréal; 2008 [consultation le 8 novembre 2010]. PDF téléchargeable à partir du lien : http://www.inspq.qc.ca/pdf/publications/777 _ServicesPremLignes.pdf. Publié conjointement par l'Institut national de santé publique du Québec et le Centre de recherche de l'Hôpital Charles LeMoyne.
24. Hamel M, Pineault R, Levesque JF, Roberge D, Lozier-Sergerie A, Prud'homme A, Simard B. L'organisation des services de santé de première ligne : portrait des services médicaux de première ligne à Montréal et en Montérégie. Montréal (Québec) : Institut national de santé publique du Québec; 2007 [consultation le 8 novembre 2010]. PDF téléchargeable à partir du lien : http://www.inspq.qc.ca/pdf /publications/726-OrganisationServices.pdf. Publié conjointement par la Direction de santé publique de l'Agence de la santé et des services sociaux de Montréal.

25. Nietert PJ, Wessell AM, Jenkins RG, Feifer C, Nemeth LS, Ornstein SM. Using a summary measure for multiple quality indicators in primary care: the Summary QUality InDex (SQUID). Implement Sci. 2007;2:11.

26. Feifer C, Nemeth L, Nietert PJ, Wessell AM, Jenkins RG, Roylance L, et coll. Different paths to high-quality care: three archetypes of top-performing practice sites. Ann Fam Med. 2007;5(3):233-41.

27. Lamarche PA, Beaulieu MD, Pineault R, Contandriopoulos AP, Denis JL, Haggerty J. Sur la voie du changement : pistes à suivre pour restructurer les services de santé de première ligne au Canada. Ottawa (Ontario) : Fondation canadienne de la recherche sur les services de santé; 2003.

28. Schoen C, Osborn R, Doty MM, Bishop M, Peugh J, Murukutla N. Toward higher-performance health systems: adults' health care experience in seven countries, 2007. Health Aff (Millwood). 2007;26(6):w717-34.

29. Institut canadien d'information sur la santé. Expériences vécues en soins de santé primaires au Canada. Ottawa (Ontario) : Institut canadien d'information sur la santé; (2009) [consultation le 4 mai 2010]. PDF téléchargeable à partir du lien : http://secure.cihi .ca/cihiweb/products/cse_phc_aib_fr.pdf.

30. Sanmartin C, Ross N. Experiencing difficulties accessing first-contact health services in Canada: Canadians without regular doctors and recent immigrants have difficulties accessing first-contact healthcare services. Reports on difficulties in accessing care vary by age, sex and region. Healthc Policy. 2006;1(2):103-19.
31. Nutting PA, Goodwin MA, Flocke SA, Zyzanski SJ, Stange KC. Continuity of primary care: to whom does it matter and when? Ann Fam Med. 2003;1(3):149-55.

32. Haggerty JL, Pineault R, Beaulieu MD, Brunelle Y, Gauthier J, Goulet F, et coll. Room for improvement: patients' experiences of primary care in Quebec before major reforms. Can Fam Physician. 2007;53(6):1056-7.

33. Starfield B. Primary Care: Balancing health needs, services and technology. New York: Oxford University Press; 1998.

34. Christakis DA. Continuity of care: process or outcome? Ann Fam Med. 2003;1(3):131-3. 